\title{
Temporal variability of gait in Parkinson disease: effectsof a rehabilitation programme based on rhythmic sound cues
}

\author{
Miguel Fernández del Olmo, Javier Cudeiro
}

\begin{abstract}
It has been suggested that sequential movements in patients with Parkinson's disease (PD) might be improved by the effects of external rhythmic cues. We studied spatiotemporal gait parameters and temporal gait variability in patients with PD and control subjects under different walking conditions in order to investigate whether rhythmic auditory cues could improve temporal variability. A total of 30 subjects were recruited, comprising 15 patients with idiopathic PD and 15 control subjects with no history of neurological disorder. As an indicator for temporal stability, we used the coefficient of variability $(\mathrm{CV}=$ standard deviation/mean $\times 100)$ of recorded intervals between two consecutive steps (gait variability). After control values were obtained, subjects underwent a Physical Rehabilitation Programme (PRP) consisting of a variety of motor tasks performed in combination with rhythmic sounds with different cadences. Sessions lasted $1 \mathrm{~h} /$ day, five times a week for a period of four consecutive weeks. At the end of the PRP subjects were evaluated again. Following completion of the programme the patients' coefficients of variability improved significantly for the preferred gait (gait: $t=2.950, p=0.011$ ) but were not significantly different from those obtained in control subjects (gait: $t=3.873, p=0.391$ ). These results are consistent with and extend prior studies of rhythmic auditory facilitation in PD and suggest a valuable method of improving gait timing in these patients.
\end{abstract}

Keywords: Parkinson; Gait; Sound; Rehabilitation

\section{Introduction}

Disorders of gait are common symptoms of Parkinson's disease (PD) [1], and gait performance represents one of the major determinants for independence and quality of life for these patients [2]. PD gait is characterised by a particular difficulty with the internal regulation of stride length [3], even though cadence control (steps per minute) is intact and is easily modulated under a variety of conditions [4]. Parkinson's disease subjects have a higher cadence rate than control subjects for any given velocity; however, this increased cadence is a compensation for reduced step size [4]. Associated disturbances include a forward-flexed trunk, inadequate flexion at the ankle and knee, insufficient heel strike, reduced arm swing, postural instability and asymmetric stride times for both lower limbs [5], [6], [7], [8] and [9]. Pharmacological therapy is effective in the early stages of the condition. However, with disease progression these gait disorders can increase leading to start hesitation, freezing episodes in the middle of motion, or blocks while switching from one motor task to another [10] and an increased risk of falls [11]. Studies on PD gait using EMG have demonstrated that there is maintenance of the phasing of muscle activation patterns during the stride cycle, particularly in the distal lower limb muscles, with a reduction in the amplitude of gastrocnemius activity during the stance phase [12], [13] and [14]; nevertheless, research on the temporal variability of gait, such as step to step, is limited. It has been postulated that the ability to maintain a steady gait (i.e. low stride-to-stride variability of gait cycle timing and its subphases) would be diminished in PD [15]. Furthermore, it has been observed that in subjects with PD, gait variability measures were increased two and three times compared to those observed in control subjects, and the degree of gait variability correlated with disease severity [15]. Irregular timing of steps in PD suggests a disturbance of rhythmic locomotor activity generation [16].

The exact pathophysiologic nature of Parkinsonian walking disabilities is not well understood. From previous research on normal and pathologic basal ganglia function, it has been proposed that the basal ganglia are implicated in two main roles in the control of sequential movements, primarily through their interaction with the supplementary motor area [17]. The first role is as an internal cue or trigger to enable movement sequences to be carried out without attention. The second role is its contribution to cortical 'motor set'. The basal ganglia aid in the preparation, and maintenance of motor plans in a state of readiness for action, enabling motor functions to be carried out functionally and appropriately [18]. 
A series of studies has revealed that PD patients can generate a normal gait pattern in the presence of adequate regulatory sensory stimulation. It is suggested that external sensory cues can provide the necessary trigger in PD to switch from one movement component in a movement sequence to the next and thus bypass defective internal pallidocortical projections [3] and [19], possibly via the lateral premotor cortex control of externally guided movements [20] and [21]. The most well known method of such stimulation is the use of visual cues placed on the floor at the desired step length to assist with gait initiation and execution [22]. With this, PD subjects can produce a gait pattern of normal velocity, cadence and stride length [3] and [23]. The use of attentional strategies such as mentally rehearsing the forthcoming movement during execution, and avoiding task distractions (the 'dual task' situation) also improve the gait pattern in PD [17], [22], [24] and [25]. The use of auditory cues also increases the length and cadence of the stride in PD subjects [26] and [27]. This finding has led to different intervention strategies in physical therapy to improve Parkinsonian gait by using music and rhythmic auditory cues, therapies such as Rhythmic Auditory Stimulation (RAS) [28] and metronome therapy [29].

In this study, we examined spatiotemporal gait parameters and gait variability in patients with PD and control subjects under different walking conditions, investigating the effect (in situ) of attentional strategies (remembered rhythmic auditory cues) and external cues (rhythmic auditory cues) in both groups in order to discover if this temporal variability can be improved.

\section{Methods}

\subsection{Subjects}

Thirty subjects were recruited for this study, comprising 15 patients with idiopathic PD (8 men and 7 women, aged 61.7 (SD 5.22) and 15 control subjects matched for height (11 men and 4 women, aged 63.1 (SD 4.28) with no history of neurological disorder. All subjects provided informed consent according to the declaration of Helsinki (1964). PD subjects were excluded if they had musculoeskeletal, cardiovascular or visual disturbances that affected walking ability, or a score $<20$ on the short test of mental status [30]. A fundamental requirement for inclusion in the study was the ability to walk up and down a $30 \mathrm{~m}$ walkway five times without walking aids or assistance. For PD subjects the level of functional disability was also determined by mean of the UPRDS, Hoehn and Yahr and Schawb and England scales [1], [31] and [32]. Table 1 shows the specific characteristics of PD group. Drug treatment was kept unchanged throughout the study.

Table 1. Specific characteristics of PD group

\begin{tabular}{|c|c|c|c|c|c|c|c|}
\hline Patient & Sex & Age & Years since diagnosis & STMS & Hoehn and yahr & Schwab and England (\%) & UPRDS \\
\hline A.A.M. & $\mathrm{F}$ & 56 & 5 & 25 & 2 & 90 & 24 \\
\hline M.G.V. & M & 64 & 6 & 23 & 1 & 90 & 26 \\
\hline C.B.R. & M & 63 & 4 & 28 & 2.5 & 50 & 41 \\
\hline F.M.C. & M & 62 & 6 & 25 & 1 & 90 & 23 \\
\hline M.F.R. & $\mathrm{F}$ & 64 & 6 & 32 & 2.5 & 80 & 38 \\
\hline D.R.M. & M & 65 & 8 & 33 & 2.5 & 80 & 45 \\
\hline J.E.L. & $\mathrm{F}$ & 65 & 5 & 30 & 2 & 90 & 28 \\
\hline J.C.I. & M & 70 & 15 & 21 & 1.5 & 50 & 41 \\
\hline C.S.S. & $\mathrm{F}$ & 69 & 6 & 30 & 1 & 90 & 13 \\
\hline G.P.V. & M & 69 & 19 & 28 & 2.5 & 50 & 57 \\
\hline
\end{tabular}

STMS, short test of mental status. 


\subsection{Procedure}

The patients were always examined at the peak dose effect of their antiparkinsonian medication. Patients underwent the following sequence of events-evaluation 1, four weeks of a PRP (see below), followed one day later by evaluation 2. Controls were evaluated once and did not undertake the PRP.

For the study patients walked along a $30 \mathrm{~m}$ flat walkway. To measure the temporal and spatial parameters of the footstep pattern, a specific system was developed in house. It consists of a set of switches worn as insoles inside the shoes, sending data in real time (sampling rate $1 \mathrm{~ms}$ ) by means of a small radio transmitter $(10 \times 5 \times 2 \mathrm{~cm})$, to a radio receiver connected to a computer. The system was also connected to two pairs of photoelectric cells, one placed at the beginning and one at the end of the walkway.

\subsection{Evaluation conditions and gait parameters}

For each evaluation the subjects walked under several different conditions:

(1) baseline condition, the instruction to the subject was simply to 'walk to the end of the walkway, at your preferred speed' (preferred gait).

(2) The subject had to walk to the end of the runway at their preferred speed and carry an object in one hand, whilst make repetitive movements (apposition of thumb and index finger) with the other hand (preferred gait with manual task).

(3) The instruction was to 'walk to the end of the walkway at your fastest speed' (fast gait).

(4) After listening to a cadence produced by a electrical metronome for $10 \mathrm{~s}$, they attempted to walk maintaining the same cadence. The following cadences were used: 60, 90, 120, $150 \mathrm{bpm}$ (reproduction gait task).

(5) Immediately following this, they attempted to complete the same series but in presence of auditory cues delivered by the metronome (synchronized gait task).

The dependent variables measured for each gait variable included: velocity $(\mathrm{m} / \mathrm{min})$; step length (m); cadence (steps/min) and the coefficient of variability $(\mathrm{CV})$ of recorded intervals between two consecutive steps. $\mathrm{CV}$ is an indicator of temporal variability of gait, where $\mathrm{CV}=($ standard deviation/mean $) \times 100$.

\subsection{Physical rehabilitation programme}

Sessions lasted $1 \mathrm{~h}$, five times a week for a period of four consecutive weeks. The PRP included walking under several different conditions.

(1) Gait without upper limbs movements-e.g. patients were asked to walk reproducing or in synchrony with a rhythmic auditory signal. ${ }^{1}$

(2) Gait with sequential movements of the upper limbs-e.g. patients were asked to walk reproducing or in synchrony with a rhythmic auditory signal ${ }^{1}$ while touching themselves on different parts of the body, always in the same order.

(3) Gait with upper limb rhythmic repetitive movements-e.g. patients were asked to walk reproducing or in synchrony with a rhythmic auditory signal ${ }^{1}$ while repetitively apposing thumb to index finger with one hand.

(4) Gait with upper limbs bimanual movement-e.g. patients were asked to walk reproducing or in synchrony with a rhythmic auditory signal ${ }^{1}$ while manipulating an object with two hands.

(5) Gait with upper simultaneous movement-e.g. patients were asked to walk reproducing or in synchrony with a rhythmic auditory signal ${ }^{1}$ while bowling a basketball.

\subsection{Statistical analysis}

The ' $t$ ' statistics was used to analyse the inter- and intra-group statistical differences for the parameters studied of gait at preferred velocity, with manual task and fast gait. A repeated-measures multivariant analysis of variance (MANOVA) was carried out on the mean velocity, cadence, step length and coefficient of variability to analyse the parameters of gait in presence or absence of rhythmic auditory cues in both groups. Patients vs. controls represent the between subjects factor. The within subjects factors were represented by trigger (self-cue vs. auditory-cue) and cadence (60, 90, 120, 150 bpm). When a significant interaction was detected, post-hoc analyses were performed. 


\section{Results}

\subsection{Gait at preferred velocity, with manual task and fast gait}

Table 2 shows the mean values for step length, cadence and velocity in the PD group compared with the control group under each of the three conditions (preferred gait, preferred gait with manual task and fast gait). For velocity the PD group shows values smaller than the control group with differences statistically significant for each condition $(t=2.916, p=0.01$ for preferred gait; $t=3.546, p=0.01$ for preferred gait with manual task and $t=4.570, p=0.001$ for fast gait). For step length the results were very similar, the PD group showing smaller values in all conditions $(t=2.850, p=0.01$ for preferred gait; $t=3.562, p=0.001$ for gait with manual task and $t=3.669, p=0.001$ for fast gait). However, for cadence we detected significant differences only in the fast gait task $(t=3.276, p=0.003)$ where the PD group shows values smaller than controls. These results are further represented in Fig. 1. Also, in the PD group, there was a significant decrease in mean step length when we compared preferred gait with preferred gait with manual task $(t=2.416, p=0.03)$. This was not observed in the control group.

Table 2. Mean values for step length, cadence and velocity in the Parkinson's disease group compared with the control group under each of the three conditions

\begin{tabular}{|c|c|c|c|c|c|c|}
\hline Velocity (m/min) & $66.55(10.64)$ & $77.11(9.16)$ & $65.40(9.57)$ & $77.27(8.75)$ & $87.16(15.94)$ & $\begin{array}{l}115.3 \\
(17.74)\end{array}$ \\
\hline Cadence (steps/min) & $\begin{array}{l}108.92 \\
(10.27)\end{array}$ & $\begin{array}{l}112.83 \\
(10.05)\end{array}$ & $\begin{array}{l}108.99 \\
(9.42)\end{array}$ & $\begin{array}{l}112.56 \\
(11.67)\end{array}$ & $\begin{array}{l}125.88 \\
(11.48)\end{array}$ & $143.7(17.4)$ \\
\hline Step length (m) & $0.61(0.08)$ & $0.68(0.04)$ & $0.60(0.08)$ & $0.69(0.05)$ & $0.69(0.10)$ & $0.81(0.07)$ \\
\hline $\begin{array}{l}\text { Coefficient } \\
\text { variability }\end{array}$ & $7.36(5.23)$ & $4.19(2.36)$ & $5.67(2.71)$ & $2.95(1.64)$ & $5.36(3.40)$ & 5.98 (3.68) \\
\hline
\end{tabular}

Mean velocity, cadence, step length and coefficient of variability of the patients with Parkinson's disease and the controls when performing the trials a preferred gait, preferred gait with manual task and fast gait. (Standard deviations are given in parentheses).
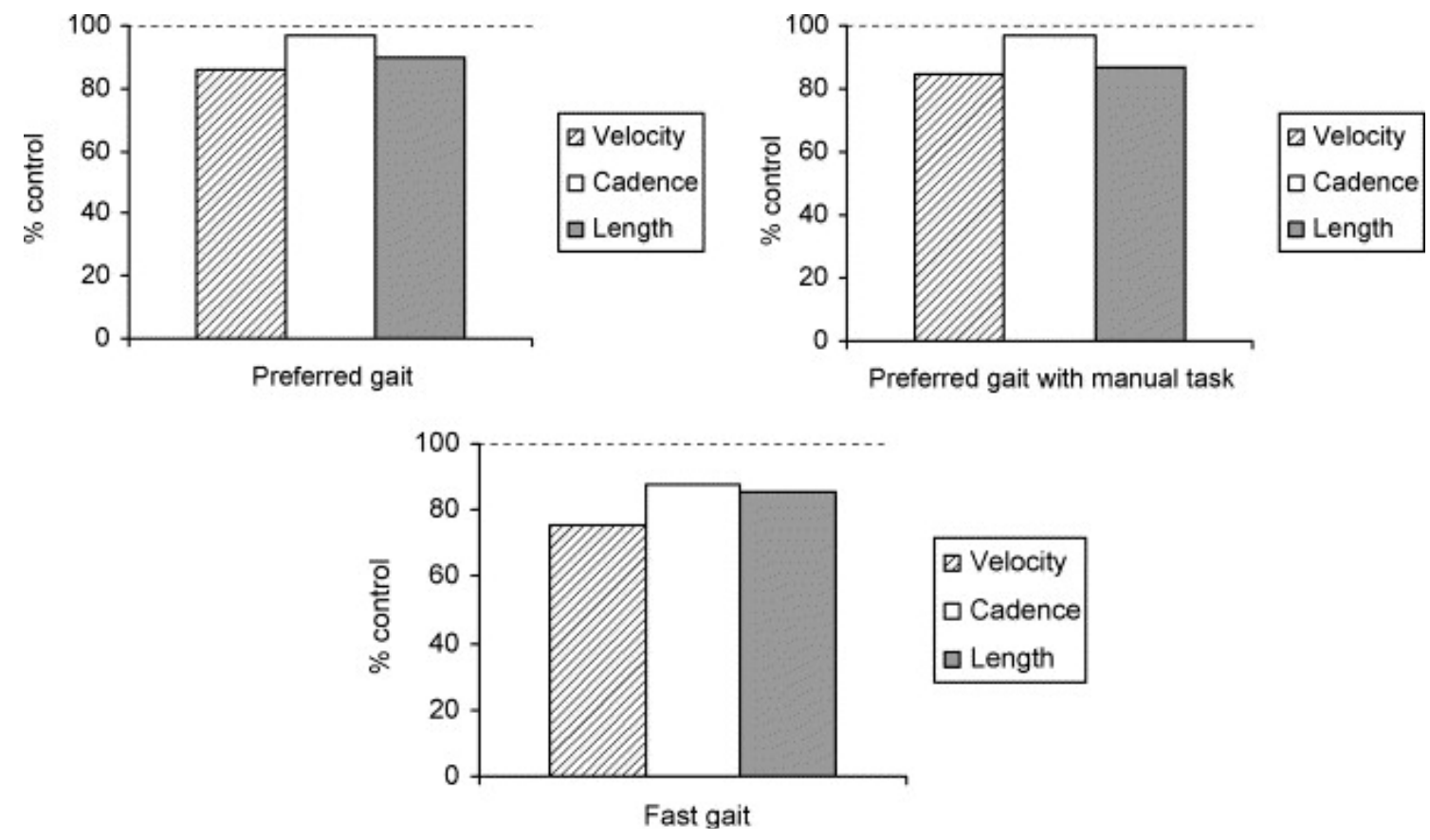

Fig. 1. Mean gait velocity, cadence and stride length for Parkinson's disease subjects expressed as a percentage of the values for matched controls. 
The recorded coefficient of variability (Table 2) for the patients was significantly greater than for controls in preferred gait $(t=2.059, p=0.049)$ and preferred gait with manual task $(t=3.244, p=0.003)$. However, there was no statistically significant difference between groups in the fast gait task. Both groups demonstrated a change in the coefficient of variability during the preferred gait with manual task but it was only significant for the control group $(p=0.020)$.

\subsection{Reproduction gait (self-cue) vs. synchronized gait (auditory-cue)}

Both groups showed an increase in mean velocity (Fig. 2) for each of the four cadences while attempting to remember auditory cues. For the 15 patients this increment was statistically significant at $60 \mathrm{bpm}(t=5.157, p=0.001)$ and $90 \mathrm{bpm}(t=3.907, p=0.002)$. For the control group the results were significant at $60 \mathrm{bpm}(t=10.223, p=0.001), 90 \mathrm{bpm}(t=6.636, p=0.001)$ and $120 \mathrm{bpm}(t=4.987, p=0.001)$.
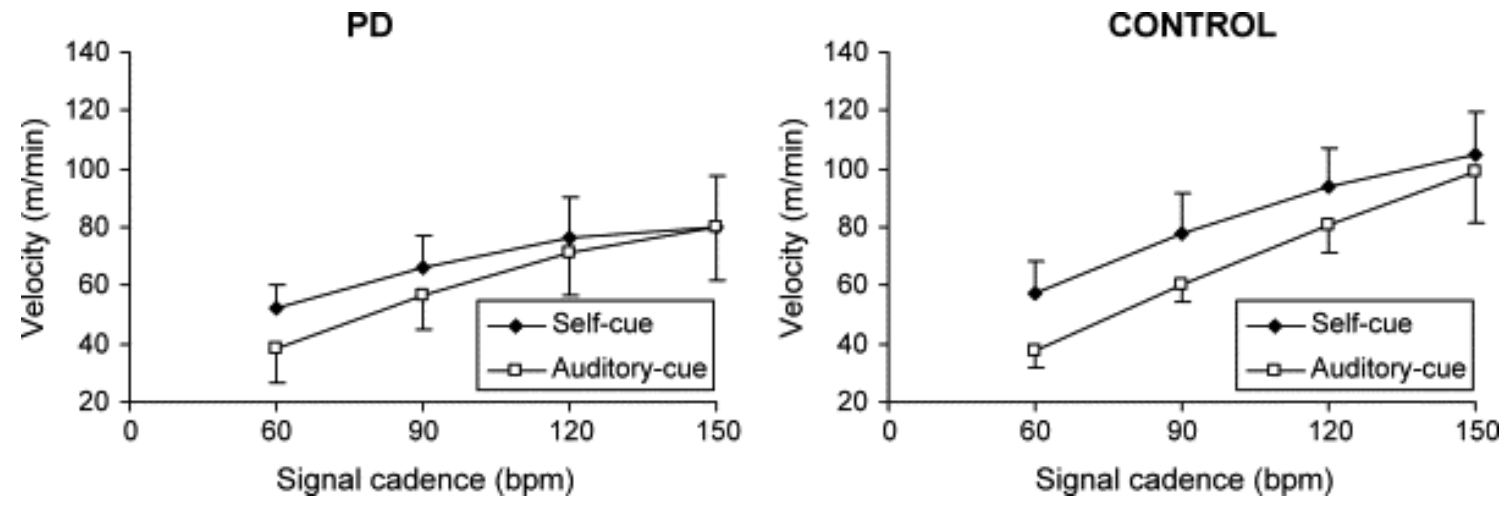

Fig. 2. Plots comparing the gait velocity performance of subjects in the presence and absence of auditory cues for the PD patients and control group.

When we studied the cadence (Fig. 3), for the PD group the mean cadence was significantly smaller in the presence of auditory cues at $60 \mathrm{bpm}(t=4.280, p=0.001)$ and $90 \mathrm{bpm}(t=3.983, p=0.001)$, there was no statistically significant difference at $120 \mathrm{bpm}$ and a significantly greater difference in the presence of auditory cues at $150 \mathrm{bmp}(t=2.757, p=0.015)$. For the control group the mean cadence was significantly smaller in the presence of auditory cues at $60 \mathrm{bpm}(t=10.138, p=0.000), 90 \mathrm{bpm}(t=5.506, p=0.004)$ and $120 \mathrm{bpm}(t=3.525, p=0.004)$, there was no statistically significant difference at $150 \mathrm{bpm}$.
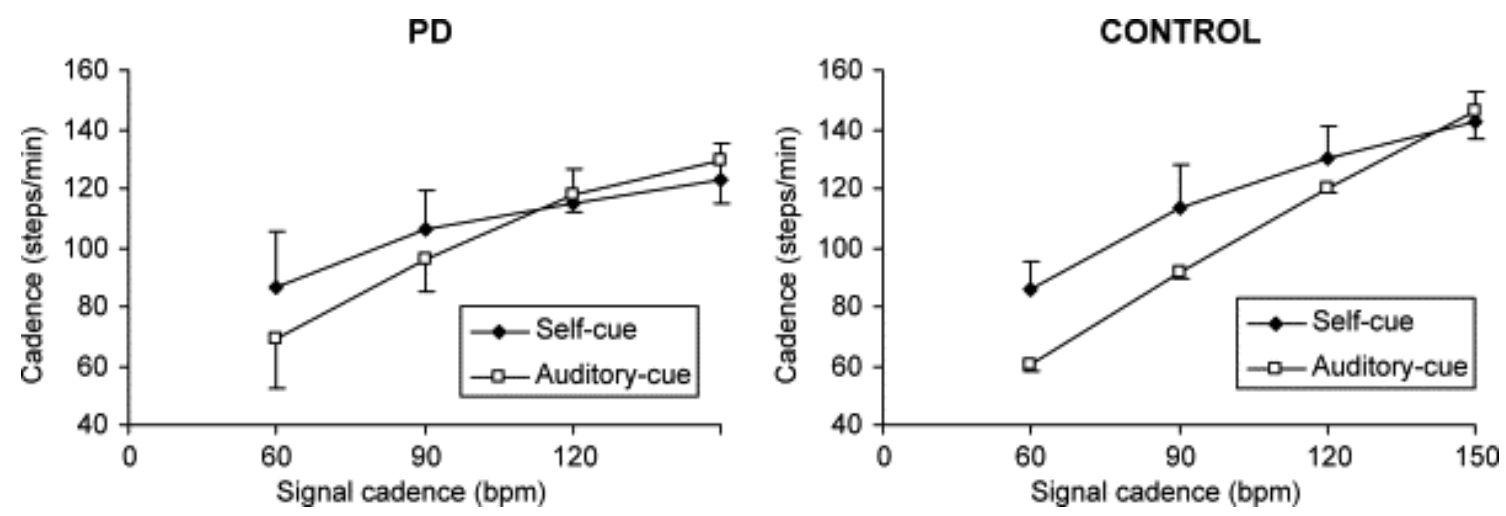

Fig. 3. Plots comparing the gait cadence performance of subjects in the presence and absence of auditory cues the PD patients and control group. 
For the step length (Fig. 4) there was a decrease in both groups for each of the four cadences in the presence of auditory cues. The multiple analysis of variance (MANOVA) showed a significant effect for the factor 'trigger' (self-cue vs. auditory-cue) for PD group $(F=11.434, p=0.004)$ and for control group $(F=6.637, p=0.024)$.
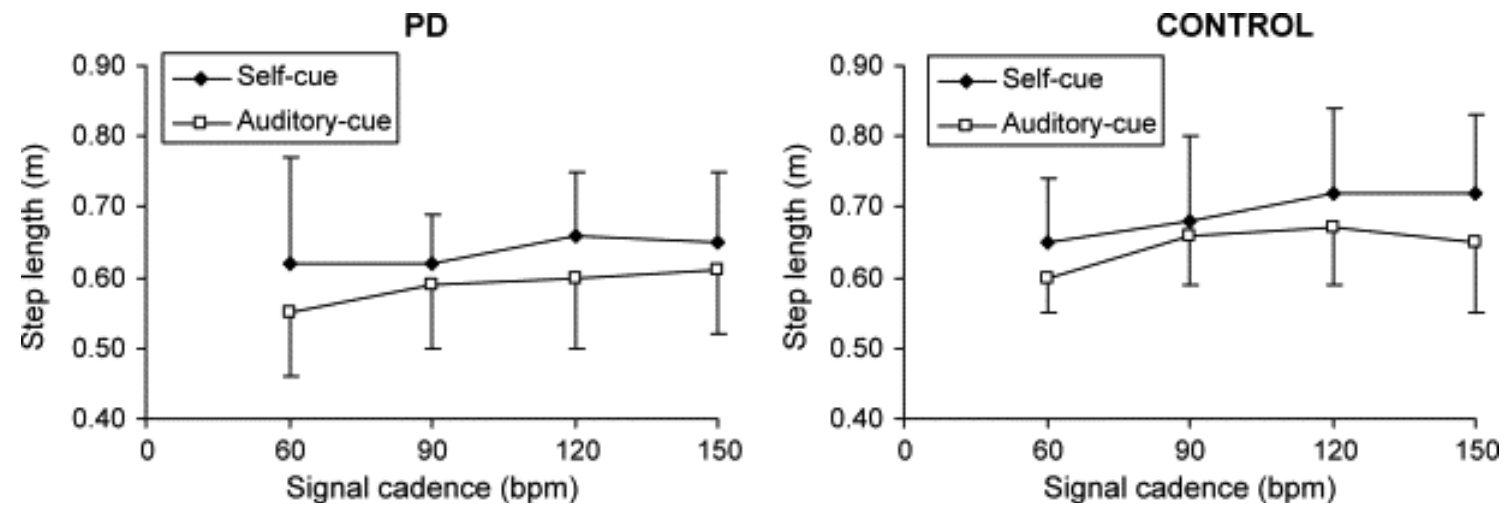

Fig. 4. Plots comparing the step length performance of subjects in the presence and absence of auditory cues for the PD patients and control group.

There were no statistically significant differences in the coefficient of variability between self-cue and audible cue for any signal cadence or for any group (Fig. 5).
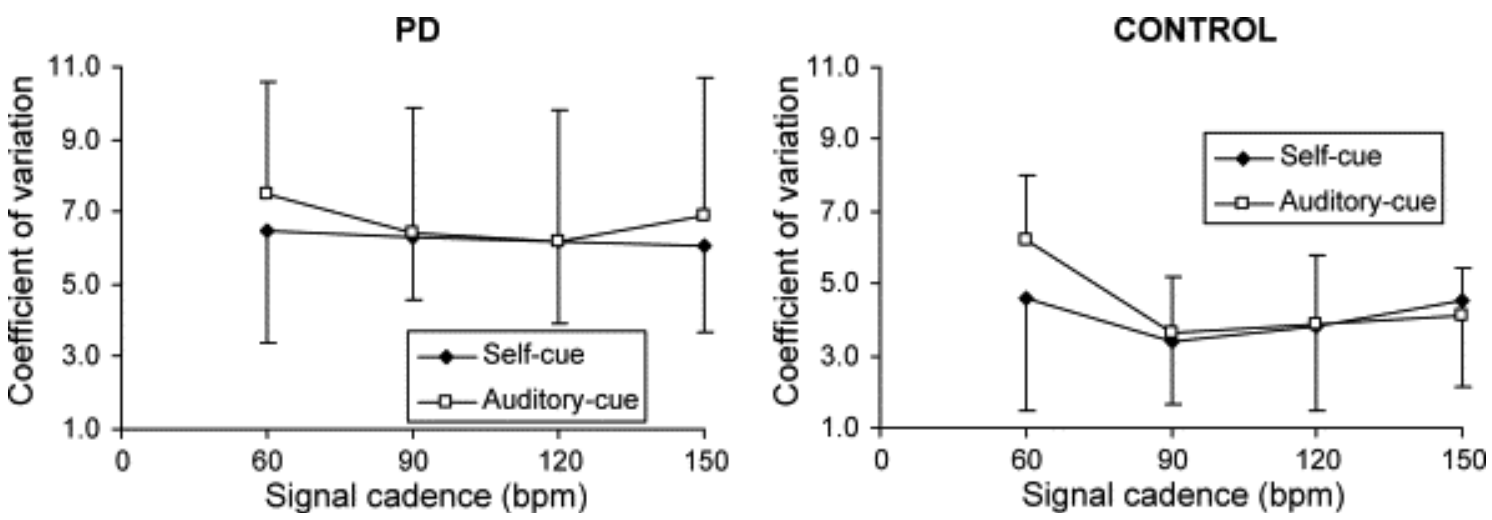

Fig. 5. Plots comparing the coefficient of variability performance of subjects in the presence and absence of auditory cues for the $\mathrm{PD}$ patients and control group.

3.3. The preferred gait, gait with manual task and fast gait before vs. after the Physical Rehabilitation Program

Following the completion of the PRP, patient results showed an improvement in the spaciotemporal gait parameters: velocity (m/min); step length $(\mathrm{m})$; cadence (steps/min) for preferred gait, preferred gait with manual task and fast gait ( Table 3). Although these parameters clearly improved towards normal values, this improvement was not statistically significant.

However, after the PRP the patients' coefficient of variability improved significantly $(t=2.950$, $p=0.011$ ) for the baseline condition (preferred gait). Surprisingly, the new values of CV recorded for the patients did not statistically differ from those obtained for control subjects $(t=3.873, p=0.391)$ ( Fig. 6). For the other tasks (preferred gait with manual task and fast gait) the CV change was not statistically significant after the PRP. 
Table 3. Gait parameters after the physical rehabilitation program was completed

\begin{tabular}{|c|c|c|c|c|c|c|}
\hline \multirow[t]{2}{*}{ Parameter } & \multicolumn{2}{|l|}{ Preferred gait } & \multicolumn{2}{|c|}{$\begin{array}{l}\text { Preferred gait with manual } \\
\text { task }\end{array}$} & \multicolumn{2}{|l|}{ Fast gait } \\
\hline & Before & After & Before & After & Before & After \\
\hline Velocity (m/min) & $66.55(10.64)$ & $69.03(10.01)$ & $65.40(9.57)$ & $66.76(10.46)$ & 87.16 (15.94) & 90.15 (16.08) \\
\hline Cadence (steps/min) & $\begin{array}{l}108.92 \\
(10.27)\end{array}$ & $\begin{array}{l}110.67 \\
(10.36)\end{array}$ & $\begin{array}{l}108.99 \\
(9.42)\end{array}$ & $\begin{array}{l}109.51 \\
(11.48)\end{array}$ & $\begin{array}{l}125.88 \\
(11.48)\end{array}$ & $\begin{array}{l}128.36 \\
(10.43)\end{array}$ \\
\hline Step length (m) & $0.61(0.08)$ & $0.62(0.08)$ & $0.60(0.08)$ & $0.61(0.08)$ & $0.69(0.10)$ & $0.69(0.11)$ \\
\hline $\begin{array}{l}\text { Coefficient } \\
\text { variability }\end{array}$ & $7.36(5.23)$ & $5.17(3.49)$ & $5.67(2.71)$ & $6.68(4.55)$ & $5.36(3.40)$ & $6.46(3.88)$ \\
\hline
\end{tabular}

Mean velocity, cadence, step length and coefficient of variability of the patients with Parkinson's disease when performing preferred gait, preferred gait with manual task and fast gait before and after the physical rehabilitation programme (Standard deviations are given in parentheses).

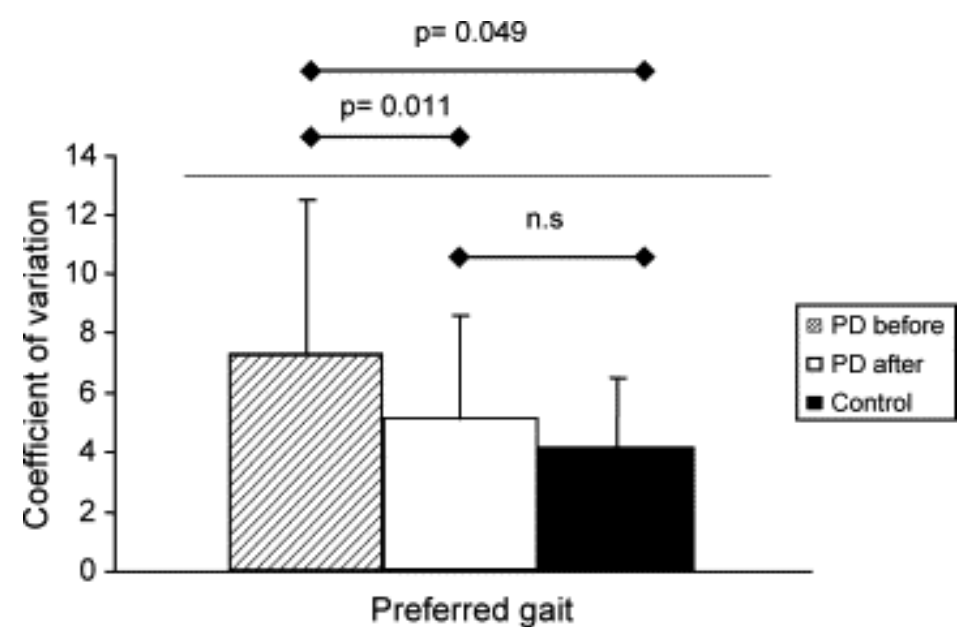

Fig. 6. Mean and standard deviation of coefficient of variability before and after the program in PD patients compared to the control group.

\section{Discussion}

\subsection{Preferred gait, preferred gait with manual task and fast gait}

Our results show that the patient group demonstrated a shorter step length and lower velocity compared with the control group under each of the three conditions (preferred gait, preferred gait with manual task and fast gait). This is in agreement with previous studies [3] and [33].

The fast gait results suggest that the most restrictive spatiotemporal parameter for the PD group is the ability to achieve a higher step cadence. When the patients walked using preferred gait their step length was $89 \%$ and cadence $96 \%$ of the values obtained for the controls. However, when the patients walked at fast gait their step length was reduced to only $85 \%$ of controls but, remarkably, the cadence dropped to $85 \%$. The value of the cadence was significantly different from that of the control group. Similar findings have been obtained by others [27]; nevertheless, previous studies [34] have shown that PD patients were able to perform higher step cadence while holding onto a rail, a condition we did not reproduce. We suggest that the ability to perform high step cadence in the absence of support is reduced in patients with PD. This does not contradict the opinion that the ability to generate a normal stepping pattern is not lost in PD. We believe that in the presence of visual cues the patients could maintain normal step length and while holding onto a rail they are able to achieve a high cadence of step [33].

Our data clearly demonstrate that during the performance of a secondary motor task the PD group displayed a deterioration in the length of the step during the preferred gait, while maintaining the cadence values. This did not occur the control group. This is in agreement with previous studies [17], [22], 
[23] and [24] and suggests that patients with PD experience considerable difficulty when they are required to walk while simultaneously performing a task with the upper limbs.

Temporal variability, was clearly impaired in the PD group during the preferred gait and preferred gait with manual task. These results reinforce the findings of other groups [15] and [16]. On the other hand, it has been suggested that the inter-step time and the step length are under the same control mechanism [35]. However, in our study the impairment in the step length in the PD group was not directly related to an impairment of temporal variability since in the preferred gait with manual task the marked deterioration was not due to an increment in the coefficient of variability. More work is required to separate the effects of these parameters.

\subsection{Reproduction gait (self-cue) vs. synchronized gait (auditory-cue)}

In this study, the general effect of the presence of auditory cues led to a decrease in the main cinematic parameters that were studied (velocity, length and cadence of walking). These results contrast with several findings reported by other groups [26] who observed a faster walking pattern when comparing the effects of either visual or auditory cues on walking parameters, although only auditory cues led to a increased stride length and its cadence, whereas visual cues led to a increased stride length but a slower stride cadence. However, it should be explained that a diminished step cadence in the presence of auditory cues at 60 and $90 \mathrm{bpm}$ was expected, due to the higher cadence performed by subjects when walking in the absence of auditory cues at these frequencies.

Rhythmic auditory stimulation facilitates locomotive function in patients with PD, as has been demonstrated [27]. It was observed that PD patients increase their maximal velocity, in presence of RAS, through a faster cadence and longer stride length. In our study, just three out of 15 PD patients showed a higher velocity when performing in the presence of an auditory cue. Two of them conquered this increase through a higher cadence but decreasing step length, and only one of them achieved this higher velocity increasing both cadence and step length. A higher step cadence was associated with a reduced step length and velocity for the rest of the patients and control subjects.

We have observed that there are differences in cadence either with or without auditory cues between groups when walking at high frequencies, 120 and $150 \mathrm{bpm}$. This supports our hypothesis that the impairment when trying to perform walking at high velocity has to do with stride length and the inability to generate high walking frequencies. This does not argue against the idea that the normal walking pattern in PD patients is still present [3] and [4], and it can be demonstrated in the presence of appropriate sensorial information. It has even, been suggested that disorders in stride length are likely linked to a deficit in the performance of fast movements in patients with PD [36].

Contrary to what we expected, the temporal stability when walking did not improve in the presence of an auditory cue in any group. This goes against the literature, which demonstrates that the presence of an appropriate sensorial cue (visual, auditory, etc.) leads to an improvement in movement timing, and in the kinetic movement parameters of walking [28]. Moreover, the worst temporal stability occurred in the presence of an external cue for some frequencies. This might be due to the demands that the presence of the auditory cue imposes on a fully automated movement. This signal requires adjusting every step in order to achieve good synchronization; this adjustment involves a number of muscle groups each with their own specific pattern of activation. Walking with no auditory cue would lead to a less consistent walk; as there is no feedback from the signal, it is not necessary to adjust every step to the signal. It has been demonstrated that the execution of ballistic rapid movements is more difficult in the presence of an auditory cue pacing the movement rhythm than in absence of one, in both patients with PD and control subjects [37]. As possible explanation of this phenomenon it was proposed that the presence of the external cue increased the difficulty of the task, because subjects had to perform the movement at maximal velocity with much precision in synchrony with the auditory cue [37]. In our study, it is notable that the worst temporal stability, in both groups, occurred for certain frequencies in presence of auditory cues, which is in agreement with some studies [16], where PD patients and control subjects showed deficits in the regulation of the stride when walking at frequencies $20 \%$ less than their preferred walk.

In conclusion, several findings can be pointed out: (a) there is a possible deficit when performing high step cadencies in patients with PD (at least in our patients), (b) a higher temporal variability was observed during walking in patients with PD, with or without auditory cues, (c) movement temporal variability is not improved in the presence of an auditory cue.

The last statement is in agreement with other studies [3], where it was observed that auditory cues did not help to normalize walking patterns of patients with PD, at least 'in situ'. However, several studies have confirmed the positive effects of using auditory cues as a strategy to improve gait [28] and [38] so that we cannot refrain from using auditory cues as a mean of training to improve this movement. 


\subsection{Preferred walk before vs. after programme}

We showed that upon completion of the 20-session program, PD subjects demonstrated a statistically significant improvement in temporal stability when walking at the preferred velocity. Thus, the program met its main objective. Moreover, this improvement allowed patients to obtain values similar to those of the control group. This was confirmed by the lack of statistically significant differences between groups at the end of the programme.

This improvement was observed in $60 \%$ of the patients, who represented those who had a greater degree of impairment in the beginning; the remainder had similar values to the control group in the beginning. The effect of the programme had more effect on the patients with greater impairment in the beginning.

What leads to the improvement in the temporal variability? Is it the mere repetition or the imposition of auditory rhythms? Some factors that determine temporal stability in walking have been proposed. Difficulty maintaining a cadence during a rhythmic repetitive movement (for instance walking) could be related to a deficit in the perception and reproduction of the rhythmic component of movement, and not just to the motor system; the cause of this deficit would be an alteration in the hypothetical internal timekeeper that controls the timing of movement [39]. Data obtained from patients with cerebellar changes suggests that this internal time-keeper could be used both in motor tasks and perceptive tasks [40], this leads one again to avoid considering the motor system as the only element responsible in the alteration of the timing of movement. Instability in walking can be considered as a maturity indicator of motor control, as evidenced by the fact that variability in walking decreases in children as they grow up [41] and starts to increase with age whether or not one has PD [42]. A recent study indicates that walking instability in aging is multifactorial, and both, neuropsychological status and health-related quality of life, play important and independent roles in gait instability [42]. In this study a group of 30 subjects all over the age of 72 years carried out a physical fitness programme for 6 months in order to improve their temporal stability when walking (this programme included exercises of strength, flexibility, as well as balance and aerobic endurance). Changes in stride time variability and the non-stationary index in response to the exercise intervention were variable and were not statistically significant despite of the fact that subjects had improved their physical fitness.

Impairment in the temporal stability of walking in the elderly has already been documented [43] but the finding of worse impairment in patients with PD could be explained by the deterioration of dopaminergic cells in the basal ganglia. The hypothesis of a deterioration regarding perceptive-motor aspects could be justified by the possible role of the basal ganglia in sensorial functions [44] or in functions that have to do with sensorimotor integration [45].

Walking exercises in the presence of an auditory rhythmic stimulation carried out by patients with PD have been shown to be an effective way of improving aspects related to the temporal pattern of the electric activation in certain muscles involved in walking, this improvement not being achieved in absence of an auditory cue [28]. The reason our patients improved their temporal variability after carrying out the programme can not be attributed to the mere repetition of the movement. The tasks used more often in the programme included walking at different temporal conditions through different auditory rhythms. Walking is a daily activity performed by our patients, nevertheless patients with PD had worse temporal variability than control subjects. Therefore, this improvement has to be linked to the use of auditory rhythmic cues. Walking in the presence of these external rhythmic auditory cues provides a stimulus that allows patients to have a better control over temporal aspects of walking. This control allowed patients to improve their variability when walking at their preferred speed, the most important for their quality of life, without having to use any external help.

This programme has been shown to be a valid way to improve temporal stability in walking, or in other words, to improve or re-educate the timing of walking, this feature allows us to affirm that temporal alterations in the execution of a movement are reversible, not only in healthy subjects [42], but in patients with PD.

\section{Acknowledgements}

We thank Dr K.L. Grieve for comments on the manuscript. This study was supported by Secretaria Xral, Investigación (PGIDIT02BTF13701PR), Xunta de Galicia, Spain. 


\section{References}

[1] M.M. Hoehn, M.D. Yahr. Parkinsonism: onset, progression, and mortality. Neurology, 17 (1967), pp. 427-442

[2] M. Schenkman. Physical therapy intervention for the ambulatory patient. G.I. Turnbull (Ed.), Physical therapy management of Parkinson's disease, Churchill Livingstone, New York (1992), pp. 137-192

[3] M.E. Morris, R. Iansek, T.A. Matyas, J.J. Summers. Ability to modulate walking cadence remains intact in Parkinson's disease. J Neurol Neurosurg Psychiatry, 57 (1994), pp. 1532-1534

[4] M.E. Morris, R. Iansek, T.A. Matyas, J.J. Summers. The pathogenesis of gait hypokinesia in Parkinson's disease. Brain, 117 (1994), pp. 1169-1181

[5]. E. Knutsson. An analysis of Parkinsonian gait. Brain, 95 (1972), pp. 475-486

[6]. M.P. Murray, S.B. Sepic, G.M. Gardner, W.J. Downs. Walking patterns of men with parkinsonism. Am J Phys Med, 57 (1978), pp. 278-329

[7] S.G. Bowes, P.K. Clark, A.L. Leeman, C.J. O'neill, C. Welle, P.W. Nicholson, A.A. Deshmukh, S.M. Dobbs, R.J. Dobss. Determinants of gait in the elderly parkinsonian on maintenance levodopa/carbidopa therapy. Br J Clin Pharmacol, 30 (1990), pp. 13-24

[8] O. Blin, A.M. Ferrandez, G. Serratrice. Quantitative analysis of gait in Parkinson patients: increased variability of stride length. J Neurol Sci, 98 (1990), pp. 91-97

[9] E. Ueno, N. Yanagisawa, M. Takami. Gait disorders in parkinsonism. A study with floor reaction forces and EMG. Adv Neurol, 60 (1993), pp. 414-418

[10] N. Giladi, D. McMahon, S. Przedborski, E. Flaster, S. Guillory, V.M. Kostic, S. Fahn. Motor blocks in Parkinson's disease. Neurology, 42 (1992), pp. 333-339

[11] J.F. Aita. Why patients with Parkinson's disease fall. JAMA, 247 (1982), pp. 515-516

[12] V. Dietz, J. Quintern, W. Berger. Electrophysiological studies of gait in spasticity and rigidity. Evidence that altered mechanical properties of muscle contribute to hypertonia. Brain, 104 (1981), pp. 431-449

[13]. M. Cioni, C.L. Richards, F. Malouin, P.J. Bedard, R. Lemieux. Characteristics of the electromyographic patterns of lower limb muscles during gait in patients with Parkinson's disease when OFF and ON L-Dopa treatment. Ital J Neurol Sci, 18 (1997), pp. 195-208

[14] V. Dietz, K.L. Leenders, G. Colombo. Leg muscle activation during gait in Parkinson's disease: influence of body unloading. Electroencephalogr Clin Neurophysiol, 105 (1997), pp. 400-405

[15] J.M. Hausdorff, M.E. Cudkowicz, R. Firtion, J.Y. Wei, A.L. Goldberger. Gait variability and basal ganglia disorders: stride-to-stride variabilities of gait cycle timing in Parkinson's disease and Huntington's disease. Mov Disord, 13 (1998), pp. 428-437

[16] G. Ebersbach, M. Heijmenberg, L. Kinderman, T. Trottemberg, J. Wissel, W. Poewe. Interference of rhythmic constraint on gait in healthy subjects and patients whit early Parkinson's disease: evidence for impaired locomotor pattern generation in early Parkinson's disease. Mov Disord, 14 (1999), pp. 619-625

[17] M.E. Morris, R. Iansek, J.J. Summers, T.A. Matyas. Motor control considerations for the rehabilitation of gait in Parkinson's disease. D.J. Glencross, J.P. Piek (Eds.), Motor control and sensory motor integration: issues and directions, Elsevier, Amsterdam (1995), pp. 61-93

[18] C. Robertson, K.A. Flowers. Motor set in Parkinson's disease. J Neurol Neurosurg Psychiatry, 53 (1990), pp. 583-592

[19] R. Cunnington, R. Iansek, J. Bradshaw, J.G. Philips. Movement-related potentials in Parkinson's disease. Presence and predictability of temporal and spatial cues. Brain, 118 (1995), pp. 935-950

[20] H. Mushiake, M. Inase, J. Tanji. Neuronal activity in the primate premotor, supplementary, and precentral motor cortex during visually guided and internally determined sequential movements. J Neurophysiol, 66 (1991), pp. 705-718

[21] U. Halsband, N. Ito, J. Tanji, H.J. Freund. The role of premotor cortex and the supplementary motor area in the temporal control of movement in man. Brain, 116 (1993), pp. 243-266

[22] M. Quintyn, E. Cross. Factors affecting the ability to initiate movement in Parkinson's disease. Phys Occup Ther Geriatr, 6 (1986), pp. 51-60

[23] M.E. Morris, R. Iansek, T.A. Matyas, J.J. Summers. Stride length regulation in Parkinson's disease. Normalization strategies and underlying mechanisms. Brain, 119 (1996), pp. 551-568

[24] M.P. Yekutiel, A. Pinhasov, G. Shahar, H. Sroka. A clinical trial of the re-education of movement in patients with Parkinson's disease. Clin Rehabil, 5 (1991), pp. 207-214

[25] A.L. Behrman, P. Teitelbaum, J.H. Cauraugh. Verbal instructional sets to normalise the temporal and spatial gait variables in Parkinson's disease. J Neurol Neurosurg Psychiatry, 65 (1998), pp. 580-582

[26] C.L. Richards, F. Malouin, P.J. Bedard, M. Cioni. Changes induced by l-dopa and sensory cues on the gait of parkinsonian patients. M. Woollacott, F. Horak (Eds.), Posture and gait: control mechanisms, University of Oregon Books, Eugene, OR (1992), pp. 126-129

[27] G.C. McIntosh, S.H. Brown, R.R. Rice, M.H. Thaut. Rhythmic auditory-motor facilitation of gait patterns in patients with Parkinson's disease. J Neurol Neurosurg Psychiatry, 62 (1997), pp. 22-26

[28] M.H. Thaut, G.C. McIntosh, R.R. Rice, R.A. Miller, J. Rathbun, J.M. Brault. Rhythmic auditory stimulation in gait training for Parkinson's disease patients. Mov Disord, 11 (1996), pp. 193-200

[29] W. Enzensberger, U. Oberlander, K. Stecker. Metronome therapy in patients with Parkinson disease. Nervenarzt, 68 (1997), pp. 972-977

[30] E. Kokmen, J.M. Naessens, K.P. Offord. A short test of mental status: description and preliminary results. Mayo Clin Proc, 62 (1987), pp. 281-288 
[31] S. Fahn, R.L. Elton. Members of the UPDRS development committee. ,in: S. Fahn, C.D. Marsden, D.B. Calne, M. Goldstein (Eds.), Recent developments in Parkinson's disease, vol. 2Macmillan Health Care Information, Florham Park, NJ (1987), pp. 153-163

[32] A.C. England, R.S. Schawb. Post-operative evaluation of 26 selected patients with Parkinson's disease. J Am Geriatr Soc, 4 (1954), pp. 1219-1232

[33] M.E. Morris, R. Iansek, T.A. Matyas, J.J. Summers. Temporal stability of gait in Parkinson's disease. Phys Ther, 76 (1996), pp. 763-777

[34] M. Morris, R. Iansek, T.A. Matyas, J.J. Summers. Abnormalities in the stride length-cadence relation in parkinsonian gait. Mov Disord, 13 (1998), pp. 61-69

[35] A. Gabell, U.S. Nayak. The effect of age on variability in gait. J Gerontol, 39 (1984), pp. 662-666

[36] W. Zijlstra, A.W. Rutgers, T.W. Van Weedern. Voluntary and involuntary adaptation of gait in Parkinson's disease. Gait Posture, 7 (1998), pp. 53-63

[37] T. Platz, R.G. Brown, C.D. Marsden. Training improves the speed of aimed movements in Parkinson's disease. Brain, 121 (1998), pp. 505-514

[38] M.H. Thaut, G.C. McIntosh, S.G. Prassas, R.R. Rice. Effect of rhythmic auditory cuing on temporal stride parameters and EMG patterns in normal gait. J Neuro Rehabil, 6 (1992), pp. 185-190

[39] M.A. Pastor, M. Jahanshahi, J. Artieda, J.A. Obeso. Performance of repetitive wrist movements in Parkinson's disease. Brain, 115 (1992), pp. 875-891

[40] R.B. Ivry, S.W. Keele, H.C. Diener. Dissociation of the lateral and medial cerebellum in movement timing and movement execution. Exp Brain Res, 73 (1988), pp. 167-180

[41] J.M. Hausdorff, L. Zemany, C. Peng, A.L. Goldberger. Maturation of gait dinamics: stride-to-stride varibility and its temporal organization in children. J Appl Physiol, 86 (1999), pp. 1040-1047

[42] J.M. Hausdorff, M.E. Nelson, D. Kaliton, J.E. Layne, M.J. Berstein, A. Nuernberger, M.A. Singh. Etiology and modification of gait instability in older adults: a randomized controlled trial of exercise. J Appl Physiol, 90 (2001), pp. 2117-2129

[43] H. Nagasaki, H. Itoh, K. Hashizume, T. Furuna, H. Maruyama, T. Kinugasa. Walking patterns and finger rhythm of older adults. Percept Mot Skills, 82 (1996), pp. 435-447

[44] S.R. Snider, S. Fahn, W.P. Isgreen, L.J. Cote. Primary sensory symptoms in parkinsonism. Neurology, 26 (1976), pp. 423-429

[45] R. Hassler. Striatal control of locomotion, intencional actions and of integrating and perceptive activity. J Neurol Sci, 36 (1978), pp. 187-224 